\title{
Determinants of Laplace-like Operators on Riemann Surfaces
}

\author{
J. Bolte and F. Steiner \\ II. Institut für Theoretische Physik, Universität Hamburg, Luruper Chaussee 149, \\ D-2000 Hamburg 50, Federal Republic of Germany
}

\begin{abstract}
We calculate determinants of second order partial differential operators defined on Riemann surfaces of genus greater than one using a relation between Selberg's zeta function and functional determinants. In addition, we perform a calculation of these determinants directly using Selberg's trace formula, and compare our results with previous computations which followed the latter route.
\end{abstract}

\section{Introduction}

Since Polyakov [1] introduced his geometric, covariant approach to string perturbation theory, the question of computing functional determinants of Laplace-like differential operators on compact Riemann surfaces has gained some attention. As it is well known in string perturbation theory (see e.g. $[2,4-6,10,15]$ ), the functional integral representing the string partition function may be reduced to a finite dimensional integral over moduli (or super-moduli) space, where the integrand can be expressed in terms of some of the determinants considered here.

Several authors [2-9] have evaluated the principal dependence of these determinants on Selberg's zeta function for the most interesting case of surfaces of genus greater than one. However, to our knowledge only D'Hoker and Phong $[3,4]$ completed the computations in determining the full answer for operators acting on tensor and spinor-tensor fields of arbitrary weight. In the following we present an alternative and more straightforward calculation of those determinants. The commutation relations for the relevant first order differential operators allow us to derive the spectra of the Laplace-like operators of arbitrary weight recursively from those of lowest weight. This gives us the opportunity of setting up a closed formula for the determinants of all those operators and for all genera. For the case of constant-curvature surfaces of genus greater than one a product representation of Selberg's zeta function involving determinant functions was obtained in [7-9]. This enables us to derive explicit expressions for the desired determinants, including all constants. Alternatively, we perform a direct calcula- 
tion using a trace formula for automorphic forms, following the suggestion of one of our referees. It turns out that our final expressions are in disagreement with those of D'Hoker and Phong obtained in $[3,4]$.

Our paper is organized as follows: First we review some basic facts on hyperbolic geometry, Laplace-like operators, determinants and zeta functions. We then develop the necessary tools for computing the determinants via the first method. After having evaluated them this way, we perform the trace formula calculation and then compare our result with that of D'Hoker and Phong $[3,4]$ and comment on their computation.

\section{Hyperbolic Geometry}

We will mainly adhere to the notations and conventions of $[3,4,7]$ and restate at first the basic facts of hyperbolic geometry:

On a Riemann surface $M$ with line element $d s^{2}=g_{\alpha \beta} d x^{\alpha} d x^{\beta}$, locally isothermal coordinates may be introduced, so that $g_{\alpha \beta}=e^{2 \varphi} \delta_{\alpha \beta}$. As $M$ admits a complex structure, one may change to complex coordinates $z=x^{1}+i x^{2}$ and $\bar{z}=x^{1}-i x^{2}$, implying $d s^{2}=2 g_{z \bar{z}} d z d \bar{z} ; g_{\bar{z} \bar{z}}=0=g_{z z}, g_{z \bar{z}}=\left(g^{z \bar{z}}\right)^{-1}=\frac{1}{2} e^{2 \varphi}$.

According to the uniformization theorem of Klein and Poincaré any compact Riemann surface $M$ is conformally equivalent to some constant-curvature surface $C / \Gamma$, where $C$ is the universal covering of $M$ and $\Gamma$ is some lattice group, isomorphic to the first homotopy group of $M$. We will be concerned with the case of surfaces of genus greater than one, where $C$ is the Poincaré upper-half-plane

$$
\mathscr{H}:=\{z=x+i y \mid-\infty<x<\infty, y>0\}
$$

endowed with the line element $d s^{2}=y^{-2} d z d \bar{z}$ (hence $g_{z \bar{z}}=\frac{1}{2} y^{-2}$ ) and scalar curvature $R=-2 . \quad \Gamma$ is then a hyperbolic, discrete subgroup of $P S L(2, \mathbf{R}):=S L(2, \mathbf{R}) /\{ \pm 1\}$ (also called a Fuchsian group). Since $M$ possesses a complex structure, traceless tensors of weight $n$ may be represented as (see e.g. [10]):

$$
T^{n}:=\left\{f(z) d z^{n} \mid f(z) d z^{n}=f^{\prime}\left(z^{\prime}\right) d z^{\prime n}\right\}, \quad n \in \mathbf{N}_{0} .
$$

[A prime denotes quantities with respect to new coordinates $z^{\prime}=z^{\prime}(z)$.]

If one fixes for genus $g \geqq 2$ one of the possible $2^{2 g}$ spin structures on $M, n$ will also be allowed to take half-integer values. That means, $T^{1 / 2}$ denotes the space of spinors on $M$.

Now define covariant derivative operators on $M$ :

$$
\begin{gathered}
\nabla_{n}^{z}: T^{n} \rightarrow T^{n-1}, \quad \nabla_{n}^{z}\left[f(z) d z^{n}\right]:=\left[g^{z \bar{z}} \partial_{\bar{z}} f(z)\right] d z^{n-1}, \\
\nabla_{z}^{n}: T^{n} \rightarrow T^{n+1}, \quad \nabla_{z}^{n}\left[f(z) d z^{n}\right]:=\left[\left(g_{z \bar{z}}\right)^{n} \partial_{z}\left(\left(g^{z \bar{z}}\right)^{n} f(z)\right)\right] d z^{n+1} .
\end{gathered}
$$

With these one can define the invariant, second order differential operators (henceforth called Laplace-like):

$$
\begin{array}{ll}
\Delta_{n}^{(+)}: T^{n} \rightarrow T^{n}, & \Delta_{n}^{(+)}:=-2 \nabla_{n+1}^{z} \nabla_{z}^{n}, \\
\Delta_{n}^{(-)}: T^{n} \rightarrow T^{n}, & \Delta_{n}^{(-)}:=-2 \nabla_{z}^{n-1} \nabla_{n}^{z} .
\end{array}
$$


[This implies: $\Delta_{0}^{(+)}=\Delta_{0}^{(-)}=-\Delta ; \Delta:=\frac{1}{\sqrt{g}} \partial_{\alpha} g^{\alpha \beta} \sqrt{g} \partial_{\beta}, g:=\operatorname{det}\left(g_{\alpha \beta}\right), \Delta$ being the usual Laplace-Beltrami operator on $M$, here: $\Delta=y^{2}\left(\partial_{x}^{2}+\partial_{y}^{2}\right)$.

All the $\Delta_{n}^{( \pm)}$are non-negative and self-adjoint, provided one introduces a scalar product on $T^{n}$ :

$$
\begin{aligned}
f_{1}, f_{2} \in T^{n}:\left\langle f_{1}, f_{2}\right\rangle_{T^{n}}: & =\int_{M} d^{2} z \sqrt{g}\left(g^{z \bar{z}}\right)^{n} f_{1}^{*}(z) f_{2}(z) \\
& =2^{n-1} \int_{M} d^{2} z y^{2 n-2} f_{1}^{*}(z) f_{2}(z) .
\end{aligned}
$$

One may now define spinor-tensors the following way:

Introduce $\tilde{\Gamma} C S L(2, \mathbf{R}),-1 \in \tilde{\Gamma}$, with $\tilde{\Gamma} /\{ \pm 1\}=\Gamma$. Then define a character $\chi: \tilde{\Gamma}$ $\rightarrow\{ \pm 1\}, \chi(-1)=-1$. (Since there are $2 g$ generators for $\tilde{\Gamma}$, there will be $2^{2 g}$ inequivalent ways of choosing $\chi$ on these generators, hence $2^{2 g}$ possible spin structures.)

Define the space $S(2 n)$ of automorphic forms of weight $n$ on $M$ by:

$$
S(2 n):=\left\{f: \mathscr{H} \rightarrow \mathbf{C} \mid f(\tilde{\gamma} z)=[\chi(\tilde{\gamma})]^{2 n}\left(\frac{c z+d}{|c z+d|}\right)^{2 n} f(z) ; \tilde{\gamma}=\left(\begin{array}{ll}
a & b \\
c & d
\end{array}\right) \in \tilde{\Gamma}\right\} .
$$

On $S(2 n)$ a scalar product is introduced through:

$$
f_{1}, f_{2} \in S(2 n):\left\langle f_{1}, f_{2}\right\rangle_{S(2 n)}:=2^{n-1} \int_{M} d^{2} z y^{-2} f_{1}^{*}(z) f_{2}(z) .
$$

One is now led to define self-adjoint second order differential operators acting on spinor-tensor fields:

$$
D_{2 n}:=-\Delta+2 i n y \partial_{x}=-y^{2}\left(\partial_{x}^{2}+\partial_{y}^{2}\right)+2 i n y \partial_{x} .
$$

A short calculation shows that $D_{2 n}+n(n \pm 1)$ and $\Delta_{n}^{( \pm)}$are conjugate under the following isometry:

$$
\begin{aligned}
I: T^{n} \rightarrow S(2 n), \\
f(z) d z^{n} \rightarrow y^{n} f(z) .
\end{aligned}
$$

$I^{-1}$ can thus be used to define $T^{n}$ for $n$ being half-integer.

As $\left[D_{2 n}+n(n \pm 1)\right] y^{n} f(z)=y^{n} \Delta_{n}^{( \pm)} f(z)$, one is left with the following commuting diagram:

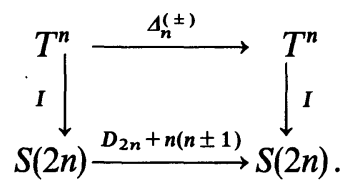

\section{Determinants, Zeta Functions, and Heat Kernels}

The operators $\Delta_{n}^{( \pm)}$(now denoted by $A$ ) are non-negative and self-adjoint on a compact manifold, thus possess a discrete spectrum with a complete set of 
eigenvectors. Denote by $0=\lambda_{0} \leqq \lambda_{1} \leqq \lambda_{2} \leqq \ldots, \lambda_{j}>\infty$ the eigenvalues of $A$. One then uses the zeta function of Minakshisundaram and Pleijel [11] (MP-zeta function) to regularize the functional determinant of $A$ :

$$
\zeta_{A}(s):=\operatorname{Tr}^{\prime} A^{-s}=\sum_{n=1}^{\infty} \lambda_{n}^{-s}, \quad \operatorname{Re} s>1 .
$$

A prime means the omission of zero-modes of $A$; the convergence for $\operatorname{Re} s>1$ follows from $\lambda_{n}=O(n), n \rightarrow \infty$, see below.

Then:

$$
\operatorname{det}^{\prime} A:=\exp \left\{-\left.\frac{d}{d s} \zeta_{A}(s)\right|_{s=0}\right\} .
$$

The trace of the heat kernel of $A$ is:

$$
\Theta_{A}(t):=\operatorname{Tr} e^{-t A}=\sum_{n=0}^{\infty} e^{-\lambda_{n} t}, \quad t>0 .
$$

This gives:

$$
\begin{gathered}
\zeta_{A}(s)=\frac{1}{\Gamma(s)} \int_{0}^{\infty} d t t^{s-1}\left[\Theta_{A}(t)-d_{0}\right], \quad \operatorname{Re} s>1, \\
d_{0}:=\operatorname{dim} \operatorname{ker} A=\text { number of zero-modes of } A .
\end{gathered}
$$

For the operators $A$ considered here, the heat kernels have the following small- $t$ asymptotics (see below): $\Theta_{A}(t)=\frac{a}{t}+O(1)$. Therefore the leading term of the asymptotic eigenvalue distribution of $A$ is given by Weyl's law:

$$
\begin{gathered}
\Theta_{A}(t)=\int_{0}^{\infty} d \lambda \frac{d N}{d \lambda} e^{-\lambda t}, \quad N(\lambda):=\#\left\{\lambda_{n} \mid \lambda_{n} \leqq \lambda\right\} \Rightarrow N(\lambda) \sim a \lambda, \quad \lambda \rightarrow \infty ; \\
\text { implying } \lambda_{n} \sim n / a, \quad n \rightarrow \infty .
\end{gathered}
$$

It is now convenient to define the determinant function $\mathscr{D}_{A}(z)$ as a Weierstrass product over its non-vanishing zeros, which converges for all $z \in \mathbf{C}$ due to Weyl's law:

$$
\begin{gathered}
\mathscr{D}_{A}(z):=\operatorname{det}^{\prime}(A+z):=\mathscr{D}_{A}(0) e^{\gamma_{A} z} \prod_{n=1}^{\infty}\left\{\left(1+\frac{z}{\lambda_{n}}\right) e^{-\frac{z}{\lambda_{n}}}\right\}, \\
\gamma_{A}:=F P \zeta_{A}(1):=\lim _{s \rightarrow 1}\left[\zeta_{A}(s)-\frac{a}{s-1}\right] .
\end{gathered}
$$

In [7-9] these functions are related to Selberg's zeta function $Z_{v}(s)$ for $-\Delta$ and $D_{1}$. These formulae will be used to compute the determinants of all $\Delta_{n}^{( \pm)}$, so we quote them here. For $-\Delta$ one has ${ }^{1}[7-9]$ (note: $\left.d_{0}=1\right)$ :

$$
\begin{aligned}
& \operatorname{det}(-\Delta+s(s-1))=s(s-1) \mathscr{D}_{\Delta}(s(s-1)) \\
& \quad=Z_{0}(s)\left\{(2 \pi)^{s} e^{-1 / 4-1 / 2 \ln 2 \pi+2 \zeta^{\prime}(-1)-s(s-1)} G(s)^{-1} G(s+1)^{-1}\right\}^{2(g-1)} .
\end{aligned}
$$

\footnotetext{
${ }^{1}$ Note the slight inconsistency in our notation, since we write $\mathscr{D}_{\Delta}$ instead of $\mathscr{D}_{-\Delta}$
} 
Here $\zeta(s)$ denotes Riemann's zeta function. For $D_{1}$ one derives [7] (note: $d_{0}=0$ ):

$$
\begin{aligned}
\operatorname{det}\left(D_{1}+s(s-1)\right) & =\mathscr{D}_{D_{1}}(s(s-1)) \\
& =Z_{1}(s)\left\{(2 \pi)^{s} e^{-1 / 4-1 / 2 \ln 2 \pi+2 \zeta^{\prime}(-1)-s(s-1)} G\left(s+\frac{1}{2}\right)^{-2}\right\}^{2(g-1)}
\end{aligned}
$$

with Barnes' double gamma function ( $\gamma=$ Euler's constant):

$$
G(z+1):=(2 \pi)^{\frac{z}{2}} e^{-\frac{z}{2}-\frac{\gamma+1}{2} z^{2}} \prod_{n=1}^{\infty}\left\{\left(1+\frac{z}{n}\right)^{n} e^{-z+\frac{z^{2}}{2 n}}\right\}
$$

and Selberg's zeta function:

$$
Z_{v}(s):=\prod_{\{\gamma\}_{p}} \prod_{k=0}^{\infty}\left\{1-(\chi(\gamma))^{v} e^{-(k+s) l(\gamma)}\right\}, \quad \operatorname{Re} s>1, v=0,1 .
$$

$\{\gamma\}_{p}$ runs over all primitive conjugacy classes in $\Gamma$ (if $v=0$ ) or $\tilde{\Gamma}$ (if $v=1$ ),

$l(\gamma)$ denotes the length of the corresponding closed geodesic.

Using Selberg's trace formula (see e.g. [12, Vol. I, p. 448] for $m=0$ ) for the operator $-\Delta$ and taking the ansatz-function $h(p)=e^{-\left(p^{2}+1 / 4\right) t}$, one can derive [9] the following small- $t$ asymptotics for $\Theta_{\Delta}(t)^{2}$ :

$$
\begin{gathered}
\Theta_{\Delta}(t)=\frac{g-1}{t} \sum_{n=0}^{N} b_{n} t^{n}+O\left(t^{N}\right), \\
b_{0}=1 ; \quad b_{n}=\frac{(-1)^{n}}{2^{2 n} n !}\left\{1+2 \sum_{k=1}^{n}\left(\begin{array}{l}
n \\
k
\end{array}\right)\left(2^{2 k-1}-1\right)\left|B_{2 k}\right|\right\}, \text { for } n \geqq 1, \\
\Theta_{\Delta}(t)=(g-1)\left\{\frac{1}{t}-\frac{1}{3}+O(t)\right\} .
\end{gathered}
$$

$B_{2 k}$ are the Bernoulli numbers.

Doing the same for $D_{1}$ (see e.g. [12, Vol. I, p. 448] for $\left.m=1\right)$, using the same $h(p)$, one obtains a similar result (the calculation is performed in Appendix A):

$$
\begin{gathered}
\Theta_{D_{1}}(t)=\frac{g-1}{t} \sum_{n=0}^{N} a_{n} t^{n}+O\left(t^{N}\right), \\
a_{n}=\frac{(-1)^{n}}{2^{2 n} n !} \sum_{k=0}^{n}\left(\begin{array}{l}
n \\
k
\end{array}\right)(-1)^{k} 2^{2 k} B_{2 k}, \text { for } n \geqq 0, \\
\Theta_{D_{1}}(t)=(g-1)\left\{\frac{1}{t}-\frac{1}{12}+O(t)\right\} .
\end{gathered}
$$

\section{Calculation of the Determinants}

Our goal is to evaluate the functional determinant of $c \Delta_{n}^{(+)}, c \in \mathbf{R}_{+}, n \in \mathbf{N}$ or $n \in \mathbf{N}_{0}+\frac{1}{2}$, since these are the relevant quantities in string theory. (We leave the value of the constant $c$ open. It then can be chosen appropriately, according to the adopted normalizations of the operators, which are not unique in the physics literature.) To perform the calculations we determine the spectra of these operators

\footnotetext{
${ }^{2}$ Again we write $\Theta_{\Delta}$ instead of $\Theta_{-\Delta}$
} 
recursively via commutation relations. For an arbitrary (spinor-) tensor $f(z) d z^{n} \in T^{n}$ one easily finds:

$$
\begin{aligned}
-\left\{\Delta_{n}^{(-)}-\Delta_{n}^{(+)}\right\} f(z) d z^{n} & =2\left\{\nabla_{z}^{n-1} \nabla_{n}^{z}-\nabla_{n+1}^{z} \nabla_{z}^{n}\right\} f(z) d z^{n} \\
& =-n R f(z) d z^{n} .
\end{aligned}
$$

Note: This formula is valid for Riemann surfaces of any genus, not necessarily restricted to the hyperbolic case $g \geqq 2$. $(R=2$ for $g=0, R=0$ for $g=1$ and $R=-2$ for $g \geqq 2$.)

Denote the eigenvalues of $\Delta_{n}^{(+)}$by $\lambda_{k}^{(n)}$ and the respective eigenvectors by $f^{(k)}(z) d z$. Then using (23) one obtains:

$$
\Delta_{n+1}^{(+)}\left\{\left(\nabla_{z}^{n} f^{(k)}(z)\right) d z^{n+1}\right\}=\left\{-(n+1) R+\lambda_{k}^{(n)}\right\}\left[\nabla_{z}^{n} f^{(k)}(z)\right] d z^{n+1} .
$$

Thus $\left[\nabla_{z}^{n} f^{(k)}(z)\right] d z^{n+1}$ is an eigenvector of $\Delta_{n+1}^{(+)}$with eigenvalue $\lambda_{k}^{(n+1)}=\lambda_{k}^{(n)}$ $-(n+1) R$. Hence the eigenvalues $\lambda_{k}^{(n)}$ are determined by those of "lowest weight" $n=0$ or $n=\frac{1}{2}$ respectively:

$$
\begin{gathered}
n \in \mathbf{N}_{0}: \lambda_{k}^{(n)}=\lambda_{k}^{(0)}-\frac{1}{2} R n(n+1), \\
n \in \mathbf{N}_{0}+\frac{1}{2}: \lambda_{k}^{(n)}=\lambda_{k}^{(1 / 2)}-\frac{1}{2} R\left(n^{2}+n-\frac{3}{4}\right),
\end{gathered}
$$

$\lambda_{k}^{(0)}$ being the eigenvalues of $\Delta_{0}^{(+)}=-\Delta$ and $\lambda_{k}^{(1 / 2)}$ those of $\Delta_{1 / 2}^{(+)}$.

In the case of genus $g \geqq 2$ these eigenvalues correspond to the continuous spectrum of the operator $D_{2 n}$ acting on the whole of $\mathscr{H}$. In addition, $D_{2 n}$ has a discrete spectrum. ${ }^{3}$ These eigenvalues can be understood physically as "Landaulevels," when one imagines $D_{2 n}$ as a Hamiltonian to describe a free particle in a magnetic field on $\mathscr{H}$. The discrete spectrum was determined e.g. in [16] for the Hamiltonian

to be

$$
H_{n}=-\Delta-2 i n y \partial_{x}+n^{2}=D_{-2 n}+n^{2}
$$

$$
E_{n}=n^{2}+\frac{1}{4}-\left(n-m-\frac{1}{2}\right)^{2}, \quad 0 \leqq m<n-\frac{1}{2} .
$$

According to Fay [14] the spectrum of $D_{2 n}$ depends only on $|n|$, thus for $n \geqq 0$ one can replace $D_{-2 n} \rightarrow D_{2 n}$. This leads to the identification

$$
\Delta_{n}^{(+)} \simeq D_{2 n}+n(n+1) \simeq H_{n}+n,
$$

which gives the discrete spectrum of $\Delta_{n}^{(+)}$as

$$
\tau_{m}^{(n)}=n^{2}+\frac{1}{4}-\left(n-m-\frac{1}{2}\right)^{2}+n=2 n m+2 n-m^{2}-m, \quad 0 \leqq m<n-\frac{1}{2} .
$$

The eigenvalues in the discrete spectrum of $\Delta_{n}^{(+)}$remain unchanged when turning to the compact surface $M=\mathscr{H} / \Gamma$. So the spectrum of $\Delta_{n}^{(+)}$on $M$ splits into the "discrete" part $\left\{\tau_{m}^{(n)}\right\}$ and the "continuous" part $\left\{\lambda_{k}^{(n)}\right\}$, which is of course also discrete on $M$, but arises from the continuous spectrum on $\mathscr{H}$. According to this splitting of the spectrum the determinants fall into a product of two contributions,

$$
\operatorname{det}^{\prime}\left(c \Delta_{n}^{(+)}\right)=\operatorname{det}^{\prime}\left(c \Delta_{n}^{(+)}\right)_{\operatorname{dis}} \operatorname{det}^{\prime}\left(c \Delta_{n}^{(+)}\right)_{\text {con }} .
$$

\footnotetext{
${ }^{3}$ We would like to thank $\mathrm{K}$. Oshima for pointing out that this part of the spectrum also contributes to the determinants and for drawing our attention to [17]
} 
A prime on det indicates that the zero-modes of the operator should be omitted.

For $g=0,1$ there is no additional "discrete" part of the spectrum and thus $\operatorname{det}^{\prime}\left(c \Delta_{n}^{(+)}\right)=\operatorname{det}^{\prime}\left(c \Delta_{n}^{(+)}\right)_{\text {con }}$.

In a first step the "continuous" part will be treated. There the above recursion relations allow us to present general formulae for this part of the desired determinants $(g \geqq 0)$ :

$$
\begin{gathered}
n \in \mathbf{N}_{0}: \operatorname{det}^{\prime}\left(c \Delta_{n}^{(+)}\right)_{\text {con }}=\operatorname{det}^{\prime}\left\{c\left(-\Delta-\frac{1}{2} R n(n+1)\right)\right\}, \\
n \in \mathbf{N}_{0}+\frac{1}{2}: \operatorname{det}^{\prime}\left(c \Delta_{n}^{(+)}\right)_{\text {con }}=\operatorname{det}^{\prime}\left\{c\left(\Delta_{1 / 2}^{(+)}-\frac{1}{2} R\left(n^{2}+n-\frac{3}{4}\right)\right)\right\} .
\end{gathered}
$$

We now again restrict ourselves to the case of hyperbolic surfaces $(g \geqq 2$, $R=-2$ ). There we use the conjugacy of $\Delta_{n}^{(+)}$and $D_{2 n}+n(n+1)$ :

$$
\begin{aligned}
n \in \mathbf{N}: \operatorname{det}\left(c \Delta_{n}^{(+)}\right)_{\text {con }} & =\operatorname{det}(c(-\Delta+n(n+1))) \\
& =c^{\zeta_{n}(0)} \operatorname{det}(-\Delta+n(n+1)) \\
& =c^{\zeta_{n}(0)} n(n+1) \mathscr{D}_{\Delta}(n(n+1)) .
\end{aligned}
$$

Note that there are no zero-modes for $n \geqq 1$. Here we introduced:

$$
\zeta_{n}(\mathrm{~s}):=\operatorname{Tr}(-\Delta+n(n+1))^{-s}=\sum_{k=0}^{\infty}\left(\lambda_{k}^{(n)}\right)^{-s} \text {. }
$$

$$
\begin{aligned}
& n \in \mathbf{N}_{0}+\frac{1}{2}: \operatorname{det}\left(c \Delta_{n}^{(+)}\right)_{\operatorname{con}}=\operatorname{det}\left(c\left(D_{1}+n(n+1)\right)\right) \\
&=c^{\tilde{\zeta}_{n}(0)} \operatorname{det}\left(D_{1}+n(n+1)\right) \\
&=c^{\tilde{\zeta}_{n}(0)} \mathscr{D}_{D_{1}}(n(n+1)), \\
& \zeta_{n}(s):=\operatorname{Tr}\left(D_{1}+n(n+1)\right)^{-s} .
\end{aligned}
$$

As explicit formulae for $\mathscr{D}_{\Delta}$ and $\mathscr{D}_{D_{1}}$ are already known [(15) and (16)], the remaining task is to compute $\zeta_{n}(0)$ and $\widetilde{\zeta}_{n}(0)$. This can be done using the small- $t$ asymptotics of the respective heat kernels. Let $\lambda_{k}$ either be an eigenvalue of $-\Delta$ or $D_{1}$ and $\Theta(t)$ be either heat kernel. Define

$$
\Theta_{n}(t):=\sum_{k=0}^{\infty} e^{-\left(\lambda_{k}+n(n+1)\right) t}=e^{-n(n+1) t} \Theta(t) .
$$

If $\zeta_{n}(s)$ now either denotes the above $\zeta_{n}(s)$ or $\widetilde{\zeta}_{n}(s)$, then:

$$
\begin{aligned}
\zeta_{n}(s) & =\frac{1}{\Gamma(s)} \int_{0}^{\infty} d t t^{s-1} \Theta_{n}(t) \\
& =\frac{1}{\Gamma(s)} \int_{0}^{\infty} d t t^{s-1} e^{-n(n+1) t} \Theta(t), \quad \operatorname{Re} s>1 .
\end{aligned}
$$

Note: $\Theta_{n}(t)=O\left(e^{-n(n+1) t}\right), t \rightarrow \infty$.

Formulae (20) and (22) show the small-t asymptotics of $\Theta(t): \Theta(t)$ $=(g-1)\left[\frac{1}{t}-b+O(t)\right]$, with $b=\frac{1}{3}$ for $A=-\Delta$ and $b=\frac{1}{12}$ for $A=D_{1}$. Therefore:

$$
\Theta_{n}(t)=(g-1)\left\{\frac{1}{t}-b-n(n+1)+O(t)\right\} .
$$


Now we can analytically continue $\zeta_{n}(s)$ to $s=0$ :

$$
\begin{aligned}
\zeta_{n}(s)= & \frac{g-1}{\Gamma(s)} \int_{0}^{1} d t t^{s-1}\left(\frac{1}{t}-b-n(n+1)\right) \\
& +\frac{1}{\Gamma(s)} \int_{0}^{1} d t t^{s-1}\left\{\Theta_{n}(t)-(g-1)\left(\frac{1}{t}-b-n(n+1)\right)\right\} \\
& +\frac{1}{\Gamma(s)} \int_{1}^{\infty} d t t^{s-1} \Theta_{n}(t) \\
= & -(g-1)\{b+n(n+1)\}+O(s) .
\end{aligned}
$$

The second and third integrals are finite in the limit $s \rightarrow 0$, thus their contribution to $\zeta_{n}(0)$ vanishes due to the factor $\frac{1}{\Gamma(s)}$.

Thus we obtain:

$$
\zeta_{n}(0)=-(g-1)\{b+n(n+1)\}, \quad b=\frac{1}{3} \text { for }-\Delta, \quad b=\frac{1}{12} \text { for } D_{1} .
$$

Inserting (15), (16), and (40) into (33) and (35) yields the final expressions for the desired determinants:

(i) $n \in \mathbf{N}$ :

$$
\begin{aligned}
\operatorname{det}\left(c \Delta_{n}^{(+)}\right)_{\text {con }}= & n(n+1) \mathscr{D}_{\Delta}(n(n+1)) \exp \left\{-(g-1)\left[\frac{1}{3}+n(n+1)\right] \ln c\right\} \\
= & Z_{0}(n+1) \exp \left\{( g - 1 ) \left[-\left(\frac{1}{3}+n(n+1)\right) \ln c+(2 n+1) \ln 2 \pi\right.\right. \\
& \left.\left.-2\left(n+\frac{1}{2}\right)^{2}+4 \zeta^{\prime}(-1)-2 \ln (G(n+1) G(n+2))\right]\right\} .
\end{aligned}
$$

Using $G(s+1)=G(s) \Gamma(s)$ and $G(1)=1$ gives:

$$
\ln [G(n+1) G(n+2)]=\ln \Gamma(n+1)+2 \sum_{k=3}^{n} \ln \Gamma(k)
$$

(empty sums are understood to be ignored).

Thus:

$$
\begin{aligned}
\operatorname{det}\left(c \Delta_{n}^{(+)}\right)_{\text {con }}= & Z_{0}(n+1) \exp \left\{( g - 1 ) \left[-\left(\frac{1}{3}+n(n+1)\right) \ln c+(2 n+1) \ln 2 \pi\right.\right. \\
& \left.\left.+4 \zeta^{\prime}(-1)-2\left(n+\frac{1}{2}\right)^{2}-2 \ln \Gamma(n+1)-4 \sum_{k=3}^{n} \ln \Gamma(k)\right]\right\} .
\end{aligned}
$$

(ii) $n \in \mathbf{N}_{0}+\frac{1}{2}$ :

$$
\begin{aligned}
& \operatorname{det}\left(c \Delta_{n}^{(+)}\right)_{\operatorname{con}}=\mathscr{D}_{D_{1}}(n(n+1)) \exp \left\{-(g-1)\left[\frac{1}{12}+n(n+1)\right] \ln c\right\} \\
& =Z_{1}(n+1) \exp \left\{( g - 1 ) \left[-\left(\frac{1}{12}+n(n+1)\right) \ln c+(2 n+1) \ln 2 \pi\right.\right. \\
& \left.\left.-2\left(n+\frac{1}{2}\right)^{2}+4 \zeta^{\prime}(-1)-4 \ln G\left(n+\frac{3}{2}\right)\right]\right\}, \\
& \ln G\left(n+\frac{3}{2}\right)=\ln G([n]+2)=\sum_{k=3}^{[n]+1} \ln \Gamma(k) ; \quad[n]=n-\frac{1}{2}: \text { integer part of } n \text {. }
\end{aligned}
$$


This yields:

$$
\begin{aligned}
\operatorname{det}\left(c \Delta_{n}^{(+)}\right)_{\operatorname{con}}= & Z_{1}(n+1) \exp \left\{( g - 1 ) \left[-\left(\frac{1}{12}+n(n+1)\right) \ln c+(2 n+1) \ln 2 \pi\right.\right. \\
& \left.\left.+4 \zeta^{\prime}(-1)-2\left(n+\frac{1}{2}\right)^{2}-4 \sum_{k=3}^{[n]+1} \ln \Gamma(k)\right]\right\} .
\end{aligned}
$$

As a general formula for both $n$ integer and half-integer one has:

$$
\begin{aligned}
\operatorname{det}\left(c \Delta_{n}^{(+)}\right)_{\text {con }}= & Z_{2(n-[n])}(n+1) \exp \left\{( g - 1 ) \left[-\left(\frac{1}{3}-\frac{1}{2}(n-[n])+n(n+1)\right) \ln c\right.\right. \\
& +(2 n+1) \ln 2 \pi+4 \zeta^{\prime}(-1)-2\left(n+\frac{1}{2}\right)^{2}-4 \sum_{3 \leqq k<n+1} \ln \Gamma(k) \\
& \left.\left.+4\left(n-\frac{1}{2}-[n]\right) \ln \Gamma(n+1)\right]\right\} .
\end{aligned}
$$

Now we study the contribution coming from the "discrete" part of the spectrum, which will be treated for both $n$ integer and half-integer together. In [16] the normalized eigenfunctions of $\Delta_{n}^{(+)}$for the "discrete" spectrum on $\mathscr{H}$ are given to be

$$
\psi_{m, k}(z)=\sqrt{\frac{(2 n-2 m-1) m !}{4 \pi k \Gamma(2 n-m)}} e^{-i k x} e^{-k y}(2 k y)^{n-m} L_{m}^{(2 n-2 m-1)}(2 k y),
$$

where the $L_{m}^{l}$ are the usual generalized Laguerre-polynomials. Thus the "discrete part" of the heat kernel on $\mathscr{H}$ is

$$
K_{n}^{\mathrm{dis}}\left(z, z^{\prime}, t\right)=\sum_{0 \leqq m<n-1 / 2} \int_{0}^{\infty} d k \bar{\psi}_{m, k}(z) e^{-c \Delta_{n}^{(+)} t} \psi_{m, k}\left(z^{\prime}\right) .
$$

The diagonal part reads

$$
\begin{aligned}
K_{n}^{\mathrm{dis}}(z, z, t)= & \frac{1}{4 \pi} \sum_{0 \leqq m<n-1 / 2} \frac{(2 n-2 m-1) m !}{\Gamma(2 n-m)} e^{-c t\left(2 n m+2 n-m^{2}-m\right)} \\
& \times \int_{0}^{\infty} \frac{d u}{u} e^{-u} u^{(2 n-2 m-1)}\left[L_{m}^{(2 n-2 m-1)}(u)\right]^{2} \\
= & \frac{1}{4 \pi} \sum_{0 \leqq m<n-1 / 2}(2 n-2 m-1) e^{-c t\left(2 n m+2 n-m^{2}-m\right)}
\end{aligned}
$$

where use has been made of the integral 7.414(3) of [13],

$$
\int_{0}^{\infty} d x e^{-x} x^{\alpha} L_{n}^{\alpha}(x) L_{m}^{\alpha}(x)=\frac{\Gamma(\alpha+n+1)}{n !} \delta_{n, m}, \quad \operatorname{Re} \alpha>0 .
$$

According to D'Hoker and Phong [3] only the "zero-length-term" in Selberg's trace formula contributes to the trace of the heat kernel on $M=\mathscr{H} / \Gamma$. This leads one to the trace of the "discrete" part of the heat kernel for $c \Delta_{n}^{(+)}$on $M$ :

$$
\begin{aligned}
\Theta_{n^{+}}^{\mathrm{dis}}(t) & =\int_{\mathscr{H} / \Gamma} \frac{d x d y}{y^{2}} K_{n}^{\mathrm{dis}}(z, z, t) \\
& =(g-1) \sum_{0 \leqq m<n-1 / 2}(2 n-2 m-1) e^{-c t\left(2 n m+2 n-m^{2}-m\right)} .
\end{aligned}
$$


Now the "discrete" part of the MP-zeta function is

$$
\begin{aligned}
\zeta_{n^{+}}^{\text {dis }}(s) & =\frac{1}{\Gamma(s)} \int_{0}^{\infty} d t t^{s-1} \Theta_{n^{+}}^{\text {dis }}(t) \\
& =(g-1) \sum_{0 \leqq m<n-1 / 2}(2 n-2 m-1)\left[c\left(2 n m+2 n-m^{2}-m\right)\right]^{-s} .
\end{aligned}
$$

Therefore one gets by differentiating and letting $s=0$ the "discrete" part of the determinants

$$
\operatorname{det}\left(c \Delta_{n}^{(+)}\right)_{\operatorname{dis}}=\exp \left\{(g-1) \sum_{0 \leqq m<n-1 / 2}(2 n-2 m-1) \ln \left[c\left(2 n m+2 n-m^{2}-m\right)\right]\right\} .
$$

The main result of this paper may thus be summarized in the following formula for the determinants of Laplace-like operators on Riemann surfaces of genus $g \geqq 2$ :

$$
\begin{aligned}
\operatorname{det}\left(c \Delta_{n}^{(+)}\right)= & Z_{2(n-[n])}(n+1) \exp \left\{( g - 1 ) \left[-\left(\frac{1}{3}-\frac{1}{2}(n-[n])+n(n+1)\right) \ln c\right.\right. \\
& +(2 n+1) \ln 2 \pi+4 \zeta^{\prime}(-1)-2\left(n+\frac{1}{2}\right)^{2}-4 \sum_{3 \leqq k<n+1} \ln \Gamma(k) \\
& +4\left(n-\frac{1}{2}-[n]\right) \ln \Gamma(n+1)+\sum_{0 \leqq m<n-1 / 2}(2 n-2 m-1) \\
& \left.\left.\times \ln \left[c\left(2 n m+2 n-m^{2}-m\right)\right]\right]\right\},
\end{aligned}
$$

which must now be compared with the expressions (3.5) and (3.6) in [3].

\section{Direct Computation via the Selberg Trace Formula}

The same result may also be obtained using a trace formula for automorphic forms, see [12, Vol. II, p. 402f.]. For simplicity we restrict ourselves to the case $c=1$ in this chapter. If then $\varrho_{k}=\frac{1}{4}+p_{k}^{2}$ are the eigenvalues of the operator $D_{2 n}$, the formula reads in the case concerning us (i.e. for compact Riemann surfaces)

$$
\begin{aligned}
\sum_{n=1}^{\infty} h\left(p_{n}\right)= & (g-1) \int_{-\infty}^{\infty} d p p h(p) \frac{\sinh (2 \pi p)}{\cosh (2 \pi p)+\cos (2 \pi n)} \\
& +\frac{1}{2} \sum_{\{\}_{p}} \sum_{k=1}^{\infty} \frac{\chi(\gamma)^{2 n k} l(\gamma)}{\sinh (k l(\gamma) / 2)} g(k l(\gamma)) \\
& +(g-1) \sum_{0 \leqq m<n-1 / 2}(2 n-2 m-1) h\left(\frac{i}{2}(2 n-2 m-1)\right), \\
& g(x):=\frac{1}{2 \pi} \int_{-\infty}^{\infty} d p h(p) e^{i p x} .
\end{aligned}
$$

Now, $h(p)=e^{-\left(p^{2}+1 / 4\right) t}$ yields the trace of the heat kernel for $D_{2 n}$ and

$$
\Theta_{\Delta_{n}^{(+)}}(t)=e^{-n(n+1) t} \Theta_{D_{2 n}}(t) .
$$

Therefore

$$
\zeta_{\Delta_{n}^{(+)}}(s)=\frac{1}{\Gamma(s)} \int_{0}^{\infty} d t t^{s-1} e^{-n(n+1) t} \Theta_{D_{2 n}}(t), \quad \operatorname{Re} s>1
$$


The trace formula gives three contributions to the trace of the heat kernel, denoted by $\Theta_{D_{2 n}}=\Theta_{D_{2 n}}^{(1)}+\Theta_{D_{2 n}}^{(2)}+\Theta_{D_{2 n}}^{(3)}$ according to their appearance in (51), which will now be treated separately. The first contribution is

$$
\Theta_{D_{2 n}}^{(1)}(t)=2(g-1) e^{-\frac{t}{4}} \begin{cases}\int_{0}^{\infty} d p p e^{-p^{2} t} \tanh (\pi p), & n \in \mathbf{N} \\ \int_{0}^{\infty} d p p e^{-p^{2} t} \operatorname{coth}(\pi p), & n \in \mathbf{N}_{0}+\frac{1}{2} .\end{cases}
$$

The Mellin-transform (52) then gives $(\operatorname{Re} s>1)$

$$
\zeta_{\Delta_{n}^{(+)}}^{(1)}(s)=2(g-1) \begin{cases}\int_{0}^{\infty} d p \frac{p \tanh (\pi p)}{\left[p^{2}+\left(n+\frac{1}{2}\right)^{2}\right]^{s}}, & n \in \mathbf{N} \\ \int_{0}^{\infty} d p \frac{p \operatorname{coth}(\pi p)}{\left[p^{2}+\left(n+\frac{1}{2}\right)^{2}\right]^{]}}, & n \in \mathbf{N}_{0}+\frac{1}{2} .\end{cases}
$$

Now the MP-zeta functions have to be analytically continued to $s=0$. This is done in Appendix B. There we show that for $n \in \mathbf{N}$

and for $n \in \mathbf{N}_{0}+\frac{1}{2}$

$$
\begin{aligned}
\left.\frac{d}{d s} \zeta_{\left.\Delta_{n}^{+}\right)}^{(1)}(s)\right|_{s=0}= & (g-1)\left\{2\left(n+\frac{1}{2}\right)^{2}-(2 n+1) \ln 2 \pi-4 \zeta^{\prime}(-1)\right. \\
& +2 \ln \Gamma(n+1)+4 \ln G(n+1)\}
\end{aligned}
$$

$$
\left.\frac{d}{d s} \zeta_{\left.\Delta_{n}^{(}\right)+(s)}^{(1)}\right|_{s=0}=(g-1)\left\{2\left(n+\frac{1}{2}\right)^{2}-(2 n+1) \ln 2 \pi-4 \zeta^{\prime}(-1)+4 \ln G\left(n+\frac{3}{2}\right)\right\} .
$$

The second contribution from the trace formula to $\Theta_{D_{2 n}}$ is

$$
\Theta_{D_{2 n}}^{(2)}(t)=\frac{e^{-t / 4}}{2 \sqrt{4 \pi t}} \sum_{\{\}_{p}} \sum_{k=1}^{\infty} \frac{\chi(\gamma)^{2 n k} l(\gamma)}{\sinh \left(\frac{k l(\gamma)}{2}\right)} e^{-\frac{k^{2} l^{2}(\gamma)}{4 t}},
$$

which gives

$$
\begin{aligned}
\zeta_{\left.\Delta_{n}^{\prime}+\right)}^{(2)}(s)= & \frac{1}{\sqrt{4 \pi}} \frac{(2 n+1)^{1 / 2-s}}{\Gamma(s)} \sum_{\{\}_{p}} \sum_{k=1}^{\infty} \frac{\chi(\gamma)^{2 n k} l(\gamma)}{\sinh \left(\frac{k l(\gamma)}{2}\right)} \\
& \times(k l(\gamma))^{s-1 / 2} K_{s-1 / 2}\left(\frac{k l(\gamma)}{2}(2 n+1)\right),
\end{aligned}
$$

where $K_{v}(z)$ denotes a modified Bessel function. Therefore

$$
\left.\frac{d}{d s} \zeta_{\Delta_{n}^{(+)}}^{(2)}(s)\right|_{s=0}=-\ln Z_{2(n-[n])}(n+1) .
$$

The third contribution can also easily be obtained,

$$
\Theta_{D_{2 n}}^{(3)}(t)=(g-1) \sum_{0 \leqq m<n-1 / 2}(2 n-2 m-1) \exp \left[-t\left(2 n m+n-n^{2}-m^{2}-m\right)\right],(60)
$$


implying

$$
\zeta_{\Delta_{n}^{(+)}}^{(3)}(s)=(g-1) \sum_{0 \leqq m<n-1 / 2}(2 n-2 m-1)\left[2 n m+2 n-m^{2}-m\right]^{-s}
$$

and

$$
\left.\frac{d}{d s} \zeta_{\Delta_{n}^{(+)}}^{(3)}(s)\right|_{s=0}=-(g-1) \sum_{0 \leqq m<n-1 / 2}(2 n-2 m-1) \ln \left(2 n m+2 n-m^{2}-m\right) .
$$

All three contributions together exactly reproduce our result obtained in Sect. 4.

\section{Conclusions}

In [3] D'Hoker and Phong computed the above considered determinants as:

$$
\begin{aligned}
& \operatorname{det}\left(\Delta_{n}^{(+)}\right)_{\mathrm{D}^{\prime} \mathrm{H.P.}}=Z_{2(n-[n])}(n+1) \\
& \quad \times \exp \left\{( g - 1 ) \left[\sum_{0 \leqq m<n-1 / 2} 2(2 n-2 m-1) \ln (2 n-m)\right.\right. \\
& \left.\left.\quad+(2 n+1) \ln 2 \pi+4 \zeta^{\prime}(-1)-2\left(n+\frac{1}{2}\right)^{2}+4(n-[n])\left(n+\frac{1}{2}\right)\right]\right\}
\end{aligned}
$$

for both $n$ integer and half-integer.

Thus our result differs from theirs. They proceeded by inserting an explicit expression for the heat kernel of $e^{-t D_{2 n}}$, which they derived from a paper by Fay [14], into Selberg's trace formula. Thus they computed a representation for the heat kernel of $\Delta_{n}^{(+)}$. Performing a Mellin-transform they obtained the MP-zeta function, which they analytically continued to $s=0$. They give:

$$
\begin{aligned}
& \operatorname{Tr} e^{-t \Delta_{n}^{(+)}}=e^{-n(n+1) t}\left\{I_{e}^{n}(t)+I_{n}(t)\right\}, \\
& I_{e}^{n}(t):=2(g-1) \sum_{0 \leqq m<n-1 / 2}(2 n-2 m-1) e^{(n-m)(n-m-1) t} \\
& +8 \pi(g-1) \frac{e^{-t / 4}}{(4 \pi t)^{3 / 2}} \int_{0}^{\infty} d b \frac{b e^{-b^{2} / 4 t}}{\sinh (b / 2)} \cosh ((n-[n]) b), \\
& I_{n}(t):=\frac{1}{4 \sqrt{\pi t}} e^{-t / 4} \sum_{\{\gamma\}_{p}} \sum_{k=1}^{\infty} \frac{[\chi(\gamma)]^{2 n} l(\gamma)}{\sinh \left(\frac{k}{2} l(\gamma)\right)} e^{-k^{2} l^{2}(\gamma) / 4 t}
\end{aligned}
$$

Note: An additional factor of two that occurred in [3] in front of the sum in $I_{e}^{n}$ has been corrected in [4]. We take the corrected version for our analysis.

According to the definition (64) their $I_{e}^{n}(t)$ is our $\Theta_{D_{2 n}}^{(1)}(t)+\Theta_{D_{2 n}}^{(3)}(t)$ and their $I^{n}(t)$ is our $\Theta_{D_{2 n}}^{(2)}(t)$ taken from Sect. 5. A comparison of the corresponding expressions shows that their $I_{e}^{n}(t)$ and $I^{n}(t)$ are both off by a factor of $\frac{1}{2}$. Besides that, the power of the character $\chi$ in $I^{n}$ should be $2 n k$. But those are the only differences from our result, as (for $n \in \mathbf{N}$ )

$$
\frac{e^{-t / 4}}{\sqrt{4 \pi} t^{3 / 2}} \int_{0}^{\infty} d b \frac{b e^{-b^{2} / 4 t}}{\sinh \left(\frac{b}{2}\right)}=\int_{-\infty}^{+\infty} d p p \tanh (\pi p) e^{-\left(p^{2}+1 / 4\right) t}
$$


which may be seen by inserting $\tanh (\pi p)=\frac{1}{\pi} \int_{0}^{\infty} d b \frac{\sin b p}{\sinh \left(\frac{b}{2}\right)}$, and (for $n \in \mathbf{N}_{0}+\frac{1}{2}$ )

$$
\frac{e^{-t / 4}}{\sqrt{4 \pi} t^{3 / 2}} \int_{0}^{\infty} d b b e^{-b^{2} / 4 t} \operatorname{coth}\left(\frac{b}{2}\right)=\int_{-\infty}^{+\infty} d p p \operatorname{coth}(\pi p) e^{-\left(p^{2}+1 / 4\right) t}
$$

which was obtained in Appendix A(69). The error in [3] thus occurs in the analytic continuation of the MP-zeta function to $s=0$.

We now would like to summarize our results as follows: In this article we have presented a method of calculating functional determinants of Laplace-like operators on constant-curvature surfaces of genus greater than one, which are interesting, e.g. in string perturbation theory. This method differs from the so far used direct trace formula calculations in that for the more involved "continuous" part of the determinants our method does not require an explicit calculation of the heat kernel for the operators on the upper half-plane. Rather our method relies on simpler considerations, concerning commutation relations of first order differential operators on the surface and the spectra of the Laplace-like operators. We also present a computation of the considered determinants that is close to the one used in [3], mainly to be able to detect the error in the calculation done in [3]. We have also presented the general formulae (31) and (32), that give expressions for the determinants on arbitrary Riemann surfaces. These can also be used for the treelevel and one-loop partition function in string perturbation theory. The well known fact in string theory that in the one-loop case $(g=1)$ the ghost determinant is in fact identical to the determinant of the Laplace operator (see e.g. $[2,4]$ ) can easily be seen from our formula (31), since in this case $R=0$ :

$$
\left(\operatorname{det}^{\prime} P_{1}^{\dagger} P_{1}\right)^{1 / 2}=\operatorname{det}^{\prime}\left(c \Delta_{1}^{(+)}\right)=\operatorname{det}^{\prime}(-c \Delta)=\frac{1}{c} \operatorname{det}^{\prime}(-\Delta) .
$$

As for the sphere $(g=0, R=2)$ the spectrum of the Laplace operator is explicitly known, the MP-zeta function for $c \Delta_{n}^{(+)}$and thus the determinant can explicitly be computed (see e.g. [15]). Alternatively the determinant may be evaluated by formula (31) (see [8]). Both expressions are found to coincide.

\section{Appendix A}

We want to determine the small- $t$ asymptotics of $\Theta_{D_{1}}(t)$, see (21):

In [12, Vol. I, p. 448], one finds a version of Selberg's trace formula concerning the operator $D_{1}$. This can be used with the ansatz-function $h(p)=e^{-\left(p^{2}+1 / 4\right) t}$.

Thus $\left(\lambda_{n}=p_{n}^{2}+\frac{1}{4}\right)$ :

$$
\begin{aligned}
\Theta_{D_{1}}(t)= & \sum_{n=0}^{\infty} h\left(p_{n}\right) \\
= & (g-1) \int_{-\infty}^{+\infty} d p p e^{-\left(p^{2}+1 / 4\right) t} \operatorname{coth}(\pi p) \\
& +\frac{1}{2 \sqrt{4 \pi t}} e^{-t / 4} \sum_{\{\gamma\}_{p}} \sum_{k=1}^{\infty} \frac{[\chi(\gamma)]^{k} l(\gamma)}{\sinh \left(\frac{k}{2} l(\gamma)\right)} e^{-k^{2} l^{2}(\gamma) / 4 t}
\end{aligned}
$$


The second contribution vanishes exponentially for $t \rightarrow 0$ and will henceforth be neglected. Using (see e.g. [13, formula 4.131(4)]:

$$
\int_{0}^{\infty} d x e^{-x} \frac{\sin a x}{\sinh x}=\frac{\pi}{2} \operatorname{coth} \frac{a \pi}{2}-\frac{1}{a}
$$

as an integral representation for $\operatorname{coth}(\pi p)$ in (59) gives:

$$
\begin{aligned}
\Theta_{D_{1}}^{(1)}(t): & =2(g-1) \int_{0}^{\infty} d p p e^{-\left(p^{2}+1 / 4\right) t} \operatorname{coth}(\pi p) \\
& =\frac{g-1}{\sqrt{\pi}} \frac{e^{-t / 4}}{\sqrt{t}}+\frac{2(g-1)}{\sqrt{\pi}} \frac{e^{-t / 4}}{t^{3 / 2}} \int_{0}^{\infty} d x \frac{x e^{-x}}{\sinh x} e^{-x^{2} / t} \\
& =\frac{2(g-1)}{\sqrt{\pi}} \frac{e^{-t / 4}}{t^{3 / 2}} \int_{0}^{\infty} d x x \operatorname{coth} x e^{-x^{2} / t}
\end{aligned}
$$

Inserting the power series: $x \operatorname{coth} x=\sum_{k=0}^{\infty} \frac{2^{2 k} B_{2 k}}{(2 k) !} x^{2 k},|x|<\pi$, yields the asymptotic expansion for $\Theta_{D_{1}}(t)$ :

$$
\begin{aligned}
\Theta_{D_{1}}(t) & =(g-1) \sum_{n=0}^{N} t^{n-1} \frac{(-1)^{n}}{2^{2 n} n !} \sum_{k=0}^{n}\left(\begin{array}{l}
n \\
k
\end{array}\right)(-1)^{k} 2^{2 k} B_{2 k}+O\left(t^{N}\right) \\
& =\frac{g-1}{t} \sum_{n=0}^{N} a_{n} t^{n}+O\left(t^{N}\right), \\
a_{n} & :=\frac{(-1)^{n}}{2^{2 n} n !} \sum_{k=0}^{n}\left(\begin{array}{l}
n \\
k
\end{array}\right)(-1)^{k} 2^{2 k} B_{2 k} .
\end{aligned}
$$

This is the expression (21).

\section{Appendix B}

In this appendix we want to derive formulae (55) and (56). To this end we define for $\operatorname{Re} s>1$ and $z \neq 0$ the functions

$$
\begin{aligned}
& I(z, s):=2(g-1) \int_{0}^{\infty} d p \frac{p \tanh (\pi p)}{\left(p^{2}+z^{2}\right)^{s}}, \\
& J(z, s):=2(g-1) \int_{0}^{\infty} d p \frac{p \operatorname{coth}(\pi p)}{\left(p^{2}+z^{2}\right)^{s}} .
\end{aligned}
$$

First, we treat $I(z, s)$ and analytically continue it to $s=0$ by a partial integration,

$$
I(z, s)=\pi \frac{g-1}{s-1} \int_{0}^{\infty} d p \frac{\left(p^{2}+z^{2}\right)^{1-s}}{\cosh ^{2}(\pi p)}
$$

Differentiation at $s=0$ gives

$$
\begin{aligned}
\left.\frac{d}{d s} I(z, s)\right|_{s=0}= & -\pi(g-1) \int_{0}^{\infty} d p \frac{p^{2}+z^{2}}{\cosh ^{2}(\pi p)}\left[1-\ln \left(p^{2}+z^{2}\right)\right] \\
= & -(g-1)\left(\frac{1}{12}+z^{2}\right)+\pi(g-1) \\
& \times \int_{0}^{\infty} d p \frac{p^{2}+z^{2}}{\cosh ^{2}(\pi p)} \ln \left(p^{2}+z^{2}\right) .
\end{aligned}
$$


To perform the integral we now differentiate with respect to $z$,

$$
\left.\frac{d}{d z} \frac{d}{d s} I(z, s)\right|_{s=0}=2(g-1) \pi z \int_{0}^{\infty} d p \frac{\ln \left(p^{2}+z^{2}\right)}{\cosh ^{2}(\pi p)} .
$$

An integral representation for the digamma function $\psi\left(z+\frac{1}{2}\right)$ (see [3, p. 17], corrected through $\ln z \rightarrow-\ln 2$ ) yields the integral in (75),

$$
\psi\left(z+\frac{1}{2}\right)=\frac{\pi}{2} \int_{0}^{\infty} d p \frac{\ln \left(p^{2}+z^{2}\right)}{\cosh ^{2}(\pi p)}
$$

therefore

$$
\left.\frac{d}{d z} \frac{d}{d s} I(z, s)\right|_{s=0}=4(g-1) z \psi\left(z+\frac{1}{2}\right) .
$$

This formula has to be integrated from $\frac{1}{2}$ to $z$,

$$
\begin{aligned}
\left.\frac{d}{d s} I(z, s)\right|_{s=0}= & \left.\frac{d}{d s} I\left(\frac{1}{2}, s\right)\right|_{s=0}+4(g-1)\left[z \ln \Gamma\left(\frac{1}{2}+z\right)\right. \\
& \left.-\int_{1 / 2}^{z} d x \ln \Gamma\left(\frac{1}{2}+x\right)\right] .
\end{aligned}
$$

Using the integral ([13], formula $6.441(4))$,

$$
\int_{0}^{z} d x \ln \Gamma(1+x)=\frac{z}{2} \ln 2 \pi-\frac{z(z+1)}{2}+z \ln \Gamma(1+z)-\ln G(1+z),
$$

and the well-known result for $z=n+\frac{1}{2}=\frac{1}{2}$ (i.e. for the operator $-\Delta$ ),

$$
\left.\frac{d}{d s} I\left(\frac{1}{2}, s\right)\right|_{s=0}=(g-1)\left[\frac{1}{2}-\ln 2 \pi-4 \zeta^{\prime}(-1)\right]
$$

we get

$$
\begin{aligned}
\left.\frac{d}{d s} I(z, s)\right|_{s=0}= & (g-1)\left[-2 z \ln 2 \pi+2 z^{2}-4 \zeta^{\prime}(-1)\right. \\
& \left.+2 \ln \Gamma\left(z+\frac{1}{2}\right)+4 \ln G\left(z+\frac{1}{2}\right)\right] .
\end{aligned}
$$

This is the result we wanted for $z=\frac{1}{2}+n, n \in \mathbf{N}$.

We now turn our attention to the function $J(z, s)$. We use $2 \operatorname{coth} 2 x=\tanh x$ $+\operatorname{coth} x$ in the definition (72) of $J$ to obtain

$$
J(z, s)=2^{1-2 s} J\left(\frac{z}{2}, s\right)+2^{1-2 s} I\left(\frac{z}{2}, s\right) .
$$

The small- $t$ asymptotics of $\Theta_{\left.\Delta_{n}^{+}\right)}(t)$ now determines $I(0, s)$ and $J(0, s)$. According to (40) this is $\left(n+\frac{1}{2}=z\right)$,

$$
\begin{aligned}
& I(0, s)=-(g-1)\left(z^{2}+\frac{1}{12}\right), \\
& J(0, s)=-(g-1)\left(z^{2}+\frac{1}{6}\right) .
\end{aligned}
$$


This may be used to obtain from (78) and (79)

$$
\begin{aligned}
\left.\frac{d}{d s} J(z, s)\right|_{s=0}= & \left.2 \frac{d}{d s} J(z / 2, s)\right|_{s=0}+2 \ln 2(g-1)\left(z^{2}+\frac{1}{6}\right) \\
& +2(g-1)\left[2 z^{2}-2 z \ln 2 \pi-4 \zeta^{\prime}(-1)\right. \\
& \left.+2 \ln \Gamma\left(z+\frac{1}{2}\right)+4 \ln G\left(z+\frac{1}{2}\right)\right] .
\end{aligned}
$$

At $z=0$ this reads, taking $G\left(\frac{1}{2}\right)=e^{3 / 2 \zeta^{\prime}(-1)} \pi^{-1 / 4} 2^{1 / 24}$ from [8],

$$
\left.\frac{d}{d s} J(0, s)\right|_{s=0}=-4(g-1) \zeta^{\prime}(-1) \text {. }
$$

To analytically continue $J(z, s)$ to $s=0$ one subtracts from the integrand in (72) the parts being divergent at the upper limit of integration and splits the region of integration,

Therefore

$$
\begin{aligned}
J(z, s)= & \frac{g-1}{s-1}-(g-1) z^{2}+2(g-1) \int_{0}^{1} d p \frac{\operatorname{coth}(\pi p)}{\left(p^{2}+z^{2}\right)^{s}} \\
& +2(g-1) \int_{1}^{\infty} d p\left[\frac{\operatorname{coth}(\pi p)}{\left(p^{2}+z^{2}\right)^{s}}-p^{1-2 s}+s z^{2} p^{-1-2 s}\right] .
\end{aligned}
$$

$$
\begin{aligned}
\left.\frac{d}{d s} J(z, s)\right|_{s=0}= & -(g-1)-2(g-1) \int_{0}^{1} d p p \operatorname{coth}(\pi p) \ln \left(p^{2}+z^{2}\right) \\
& -2(g-1) \int_{1}^{\infty} d p\left[p \operatorname{coth}(\pi p) \ln \left(p^{2}+z^{2}\right)-2 p \ln p-\frac{z^{2}}{p}\right] .
\end{aligned}
$$

To perform this integral we differentiate with respect to $z$,

$$
\left.\frac{d}{d z} \frac{d}{d s} J(z, s)\right|_{s=0}=-4(g-1) z\left\{\int_{0}^{1} d p \frac{p \operatorname{coth}(\pi p)}{p^{2}+z^{2}}+\int_{1}^{\infty} d p\left[\frac{p \operatorname{coth}(\pi p)}{p^{2}+z^{2}}-\frac{1}{p}\right]\right\} .
$$

In [13] one finds the following integral representation for the digamma function for $\operatorname{Re} z>0$ (see formula $8.361(3)$ ):

$$
\psi(z)=\ln z-\frac{1}{2 z}-2 \int_{0}^{\infty} d t \frac{t}{\left(t^{2}+z^{2}\right)\left(e^{2 \pi t}-1\right)} .
$$

Introducing $\operatorname{coth}(\pi p)$ into that integral representation and splitting the region of integration yields

$$
\psi(z)=-\frac{1}{2 z}-\int_{0}^{1} d p \frac{p \operatorname{coth}(\pi p)}{p^{2}+z^{2}}-\int_{1}^{\infty} d p\left[\frac{p \operatorname{coth}(\pi p)}{p^{2}+z^{2}}-\frac{1}{p}\right],
$$

and thus

$$
\left.\frac{d}{d z} \frac{d}{d s} J(z, s)\right|_{s=0}=4(g-1) z \psi(1+z)-2(g-1)
$$


This will now be integrated to give

$$
\left.\frac{d}{d s} J(z, s)\right|_{s=0}=\left.\frac{d}{d s} J(0, s)\right|_{s=0}+4(g-1)\left[z \ln \Gamma(1+z)-\int_{0}^{z} d x \ln \Gamma(1+x)-\frac{1}{2 z}\right] .
$$

Again the integral 6.441 (4) from [13] appears, thus

$$
\left.\frac{d}{d s} J(z, s)\right|_{s=0}=(g-1)\left[2 z^{2}-2 z \ln 2 \pi-4 \zeta^{\prime}(-1)+4 \ln G(1+z)\right],
$$

which is the desired result.

\section{References}

1. Polyakov, A.M.: Phys. Lett. 103B, 207 and 211 (1981)

2. D’Hoker, E., Phong, D.H.: Nucl. Phys. B 269, 205 (1986)

3. D'Hoker, E., Phong, D.H.: Commun. Math. Phys. 104, 537 (1986)

4. D'Hoker, E., Phong, D.H.: Rev. Mod. Phys. 60, 917 (1988)

5. Gilbert, G.: Nucl. Phys. B 277, 102 (1986)

6. Namazie, M.A., Rajeev, S.: Nucl. Phys. B 277, 332 (1986)

7. Sarnak, P.: Commun. Math. Phys. 110, 113 (1987)

8. Voros, A.: Commun. Math. Phys. 110, 439 (1987)

9. Steiner, F.: Phys. Lett. 188B, 447 (1987)

10. Alvarez, O.: Nucl. Phys. B 216, 125 (1983)

11. Minakshisundaram, S., Pleijel, A.: Can. J. Math. 1, 242 (1949)

Minakshisundaram, S.: Can. J. Math. 1, 320 (1949)

12. Hejhal, D.A.: The Selberg trace formula for $P S L(2, \mathbf{R})$, vol. I. Lecture Notes in Mathematics, vol. 548. Berlin, Heidelberg, New York: Springer 1976, vol. II. Lecture Notes in Mathematics, vol. 1001. Berlin, Heidelberg, New York: Springer 1983

13. Gradshteyn, I.S., Ryzhik, I.M.: Tables of integrals, series, and products. New York: Academic Press 1980

14. Fay, J.: J. Reine Angew. Math. 293, 143 (1977)

15. Weisberger, W.I.: Nucl. Phys. B 284, 439 (1987)

16. Grosche, C.: Ann. Phys. 187, 110 (1988)

17. Oshima, K.: Prog. Theor. Phys. 81, 286 (1988)

Oshima, K.: Phys. Rev. D 41, 702 (1990)

Communicated by S.-T. Yau

Received January 5, 1989; in revised form December 29, 1989 
